\title{
Editorial
}

\section{From chimp to human to robot, the journey goes on!}

hanges in our hands and fingers were a side-effect of changes in the shape of our feet, and bipedalism freed the hand to evolve for other purposes. Our ancestors did not have to use their forelimbs for locomotion any more and a group of chimpanzee-like apes began to throw rocks and swing clubs at adversaries, and this behaviour yielded reproductive advantages for millions of years, driving natural selection for improved throwing and clubbing prowess. The two fundamental human handgrips, first identified by J. R. Napier, and named by him as the "precision grip" and the "power grip", represent a throwing grip and a clubbing grip, thereby providing an evolutionary explanation for the two unique grips, and the extensive anatomical remodelling of the hand that made them possible. ${ }^{[1]}$

The fingers, metacarpal and carpal bones of the chimpanzee hand are elongated, but in typical primate fashion, the thumb is small, weak and relatively immobile The third and fourth metacarpals, which absorb the highest compression during knuckle-walking, are especially robust. ${ }^{[2]}$ Both proximal and middle phalanges are curved towards the palm to withstand stress from gripping limbs during arboreal locomotion. The finger tips are cone shaped and lack broad apical tufts. ${ }^{[2,3]}$ Thumb phalanges and metacarpals are slender and short and the intrinsic muscles of the thumb, underlying the thenar region of the palm, are small. ${ }^{[4]}$ With this hand, the chimpanzee's grip is designed to hang from trees a hook grip. Because the thumb is weak and short, its distal phalanx is relatively immobile and its distal pad cannot be opposed to those of the fingers, and it cannot generate a firm pinch or squeeze.

\begin{tabular}{|l|l|}
\hline \multicolumn{2}{|c|}{ Access this article online } \\
\hline Quick Response Code: & Website: \\
\hline
\end{tabular}

The human thumb in contrast is longer and stronger, the palm and fingers are shorter and the fingers have lost their curvature. The distal phalanges have gained large apical tufts that support broad, palmar, fibrofatty pads that distribute pressure during forceful grasping and whose deformation accommodates the pads to uneven surfaces. ${ }^{[2,3]}$ Apart from thickening of the fifth metacarpal and enlargement of its base, the balance of strength and robustness has shifted radially to the thumb, second and third fingers. ${ }^{[2,5]}$ The thumb metacarpal articulates with the carpals in a saddle joint, which in combination with remodelling in the metacarpal-phalangeal joint, allows its distal pad to be placed against those of the other fingers, providing full opposability ${ }^{[6]}$ The intrinsic thumb muscles are larger and three new muscles have evolved in humans to add strength and control to the thumb movements. The flexor pollicis longus muscle, absent in chimpanzees, is the most powerful thumb muscle in humans. It flexes the distal phalanx of the thumb and maintains the orientation of its pad towards the fingers against pressure. Also new are the deep head of the flexor pollicis brevis and the first volar interosseous muscle. ${ }^{[7]}$ Another unique attribute of the human hand is finger rotation. When the fingers are flexed, they rotate towards the central axis so that the fingertips can meet the tip of the thumb. The metacarpal-hamate articulation permits supination of the fourth and fifth metacarpals, whereas the heads of the second and third metacarpals allow pronation of the proximal phalanges. ${ }^{[2]}$ These modifications have given the human hand the two new grips - the precision grip and the power grip.

Taking this concept of evolution a step further, let us see how we are designing a robotic hand. HANDLE (Developmental pathway towards autonomy and dexterity in robot in-hand manipulation) is a large-scale "Integrated Project" funded by the European Union within The Seventh Framework Programme FP7, in which nine European institutions, coordinated by the Pierre and Marie Curie University of Paris (France), participate to build a robotic arm and hand. The objective of the project is to create a robotic hand that can reproduce 
the abilities and movements of a human hand in order to achieve the optimal manipulation of objects. The research carried out by the HANDLE project's partners are in the area of visual perception, motion planning, new sensors, acquisition of motor skills using artificial intelligence techniques, etc. ${ }^{[8]}$

When trying to recreate the movements of a human hand with a robotic system, there are several complex problems that must be resolved. In the first place, there is a lack of space; the human hand is incredibly complete, which makes it a challenge to try to put all of the necessary pieces into the robotic hand and to integrate all of the actuators that allow for mobility similar to that of a human hand. Again, there are currently no sensors in the market that are small enough to be integrated into the device so that it can have sensitivity similar to that of a human hand and, thus, be able to make precise movements.

Shadow Robot Company's Shadow Hand is perhaps the closest robot hand to the human hand..$^{[9]}$ It provides 24 movements, allowing a direct mapping from a human to the robot. The hand contains an integrated bank of 40 air muscles, which make it move and produce precision grip and power grip. The air muscle is a simple yet powerful device for providing a pulling force. It behaves in a very similar way to a biological muscle. When actuated with a supply of compressed air, it contracts by up to $40 \%$ of its original length. The air muscles are compliant, which allows the hand to be used around soft or fragile objects. This hand can be fitted with touch sensing on the fingertips, offering sensitivity sufficient to detect a single small coin or manipulate delicate objects such as fruit and eggs.
Finally, although the researchers may manage to make a perfect robot from the mechanical and sensorial point of view, without 'intelligence' elements, the device will not be able to function autonomously nor adapt its movements and control to the characteristics of the objects, such as their geometry, texture, weight or use. Therefore, this only goes to suggest that evolution of the hand alone did not help the human species. The associated development of the human brain was invaluable.

\section{Surajit Bhattacharya \\ Editor, Indian Journal of Plastic Surgery \\ Sr. Consultant Plastic Surgery, \\ Sahara Hospital, Lucknow, India. \\ E-mail: surajitb@sanchanet.in}

\section{REFERENCES}

1. Young RW. Evolution of the human hand: The role of throwing and clubbing. J Anat 2003;202:165-74.

2. Susman RL. Comparative and functional morphology of hominoid fingers. Am J Phys Anthropol 1979;50:215-36.

3. Napier JR. Studies of the hands of living primates. Proc Zool Soc London 1960;134:647-57.

4. Marzke MW. Evolution of the hand and bipedality. In: Lock A, Peters A, editors. Handbook of Human Symbolic Evolution. Oxford: Oxford University Press; 1992. p. 126-54.

5. Marzke MW, Shackley MS. Hominid hand use in the Pliocene and Pleistocene: Evidence from experimental archaeology and comparative morphology. J Hum Evol 1986;15:439-60.

6. Napier JR. The form and function of the carpo-metacarpal joint of the thumb. J Anat 1955;89:362-9.

7. Susman RL. Fossil evidence for early hominid tool use. Science 1994;265:1570-3.

8. Available from: http://www.handle-project.eu/

9. Available from: http:/www.shadowrobot.com

How to cite this article: Bhattacharya S. From chimp to human to robot, the journey goes on!. Indian J Plast Surg 2011;44:171-2.

Announcement

\section{“QUICK RESPONSE CODE” LINK FOR FULL TEXT ARTICLES}

The journal issue has a unique new feature for reaching to the journal's website without typing a single letter. Each article on its first page has a "Quick Response Code". Using any mobile or other hand-held device with camera and GPRS/other internet source, one can reach to the full text of that particular article on the journal's website. Start a QR-code reading software (see list of free applications from http://tinyurl.com/yzlh2tc) and point the camera to the QR-code printed in the journal. It will automatically take you to the HTML full text of that article. One can also use a desktop or laptop with web camera for similar functionality. See http://tinyurl.com/2bw7fn3 or http://tinyurl.com/3ysr3me for the free applications. 